\title{
SPEECH SEPARATION BY KURTOSIS MAXIMIZATION
}

\author{
James P. LeBlanc \\ Phillip L. De Lè̀n
}

Klipsch School of ECE

New Mexico State University

Las Cruces, NM USA

\begin{abstract}
We present a computationally efficient method of separating mixed speech signals. The method uses a recursive adaptive gradient descent technique with the cost function designed to maximize the kurtosis of the output (separated) signals. The choice of kurtosis maximization as an objective function (which acts as a measure of separation) is supported by experiments with a number of speech signals as well as spherically invariant random processes (SIRP's) which are regarded as excellent statistical models for speech. Development and analysis of the adaptive algorithm is presented. Simulation examples using actual voice signals are presented.
\end{abstract}

\section{INTRODUCTION}

We address the speech separation problem. Making some assumptions on the statistics of the voice signals we use higher order statistics to separate mixed voices. The use of higher-order statistics is not new to the source separation problem, see ([2] [1], [9], [1], [8] for example). Many of these methods are applied to digital communications signals which inherently belong to a different statistical class than speech signals. Specifically, many such adaptive algorithms used on digital communications signals use the fact that the sequences are sub-Gaussian ${ }^{1}$. We note that there are separtation algorithms designed for use with speech signals (notably [11] and [10]). Some methods are computationally intensive, necessitating correlation matrix estimation and eigen-decompositions or polyspectra estimations. As an alternative, the speech separation developed herein is designed to be computationally efficient. We exploit the fact that speech signals are super-Gaussian (i.e. have high kurtosis). Noting this, we may adopt similar strategies using higher order statistics after appropriate modification.

A fundamental idea of many blind separation and blind equalization schemes in the digital communications setting is to note that the sum of sub-Gaussian processes (as occurs with mixing and intersymbol interference) results in a process with statistics that differ from the original process(es). More specifically, the mixture "looks more" Gaussian than the originals. In [7] one finds an excellent discussion of developing measures of Gaussianity. With such a measure, one may construct a cost function, and associated adaptive gradient descent algorithm which minimizes this Gaussianity and results in source separation or intersymbol interference reduction (for the sub-Gaussian digital communications signals). Many possible measures are possible. One which appears quite often is kurtosis.

\footnotetext{
${ }^{1}$ The term sub-Gaussian may have different meanings among different communities. Here it is used to denote processes having a kurtosis less than the kurtosis of a Gaussian.
}

The relation of kurtosis to one of the the more popular blind equalization algorithm known as the Constant Modulus Algorithm (CMA) [8] or Godard Algorithm [6] is discussed in [5]. For the separation of speech signals, we modify a source separation algorithm recently proposed for digital communications (sub-Gaussian) signals in [3] which uses the CMA (or Godard) error function. This modification adjusts for the differing statistics between digital communication signals and voice signals.

\section{PROBLEM SETTING}

The generic two signal separation problem is shown in Figure 1. Two sources $s_{0}$ and $s_{1}$ are mixed through mixing matrix $\mathbb{A}$, resulting in received signals $x_{0}$ and $x_{1}$. The mixing relation is denoted,

$$
X=\mathbb{A} S
$$

where $X=\left[\begin{array}{ll}x_{0} & x_{1}\end{array}\right]^{t}$ and $S=\left[\begin{array}{ll}s_{0} & s_{1}\end{array}\right]^{t}$.

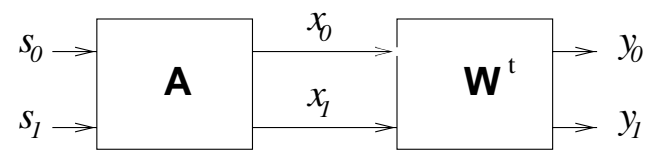

\section{Figure 1. Separation Block Diagram}

The goal is to separate out the $s_{0}$ and $s_{1}$ components present in the mixed signals $x_{0}$ and $x_{1}$ through the use of matrix $\mathbb{W}^{t}$. Clearly, $\mathbb{W}^{t}=\mathbb{A}^{t-1}$ achieves the desired result (assuming $\mathbb{A}$ is invertible) but, $\mathbb{A}$ is typically unknown. In the blind problem, $\mathbb{W}$ (or similarly $\mathbb{A}$ ) must be estimated from knowledge only of the mixture $X$. The second order statistics of $X$ (i. e. the autocorrelation matrix $\mathbb{R}_{X X}$ ) do not provide enough information. For this reason higher order statistics are often considered. However, the use of higher order statistics often requires further assumptions on the distributions of $S$. In the blind separation problem (as well as the blind equalization problem), the source distributions are typically considered to be sub-Gaussian in the sense that their kurtosis is below that of a Gaussian. The kurtosis ${ }^{2}$ of a zero mean random variable $x$ is defined as the dimensionless, scale invariant quantity,

$$
\kappa_{\mathrm{X}}=\frac{\mathrm{E}\left\{x^{4}\right\}}{\left\{\mathrm{E}\left\{x^{2}\right\}\right\}^{2}}
$$

where $\mathrm{E}\{\bullet\}$ is the expectation operator. For any random variable we have $\kappa \geq 1$, and for a Gaussian distribution $\kappa=$

\footnotetext{
${ }^{2}$ The reader is cautioned that some texts define kurtosis a bit differently as $\kappa_{\mathrm{X}}=\frac{\mathrm{E}\left\{x^{4}\right\}}{\left(\mathrm{E}\left\{x^{2}\right\}\right)^{2}}-3$. We shall however follow the definition above as found in [13].
} 
3. Distributions with $\kappa<3$ are considered sub-Gaussian (or platykurtic), and those with $\kappa>3$ are labelled as superGaussian (or leptokurtic).

\section{SEPARATION BY KURTOSIS}

\subsection{Communications Signals}

An interesting feature of kurtosis is now noted. Let $u_{0}$ and $u_{1}$ be two independent, identically distribute (iid), zero mean random variables with kurtosis $\kappa_{\mathrm{U}}$. Let $w=u_{0}+u_{1}$ and consider $\kappa_{\mathrm{W}}$. It can be shown that $\kappa_{\mathrm{W}}$ is always closer to 3 , than $\kappa_{\mathrm{U}}$. More specifically,

$$
\begin{aligned}
& \text { if } \kappa_{\mathrm{U}}<3 \text { (platyurtic), then } \kappa_{\mathrm{W}}>\kappa_{\mathrm{U}} \\
& \text { if } \kappa_{\mathrm{U}}>3 \text { (leptokurtic), then } \kappa_{\mathrm{W}}<\kappa_{\mathrm{U}}
\end{aligned}
$$

Since, digital communications signal are typically leptokurtic. Given two iid sources, the resulting mixture will have a higher kurtosis. Thus, a logical separation strategy is to minimize the kurtosis, which in effect, is exactly what CMA does. In [3] an iterative separation algorithm from digital communications signals utilizing the CMA error function is presented as

$$
\mathbb{W}_{n+1}=\mathbb{W}_{n}-\mu \nabla \mathbb{W}(\phi(\mathbb{W}))
$$

where $\mu$ is the small adaptive stepsize, and $\nabla_{\mathbb{W}} \phi(\mathbb{W})$ denotes the gradient of $\phi$. Given as

$$
\phi(\mathbb{W})=\sum_{i=1}^{N} \mathrm{E}\left\{\left(y_{i}^{2}-1\right)^{2}\right\}-\ln (\operatorname{det}|\mathbb{W}|)
$$

in which the first term is as the CMA cost function, while the second term associates a cost to duplicating a source at the output $Y$. The existence of the CMA cost term can be associated with a gradient descent algorithm performing a kurtosis minimization on the output $Y$. In light of (3), such kurtosis minimization agrees with source separation.

\subsection{Speech Signals}

We adopt a kurtosis-based strategy for separating speech signal by observing that speech signal are leptokurtic (in contrast to the typcially platykurtic communications signals). In light of (4), we choose the adaptation objective to be kurtosis maximization. The adaptive algorithm becomes (ignoring for the moment the desire to prevent duplicate sources at output),

$$
\mathbb{W}_{n+1}=\mathbb{W}_{n}+\mu \nabla_{\mathbb{W}}\left(\kappa_{\mathrm{Y}}(\mathbb{W})\right)
$$

where $\mu$ is the small adaptive stepsize, and $\nabla_{\mathbb{W}} \kappa_{Y}(\mathbb{W})$ denotes the gradient of the kurtosis of the outputs $Y$. For the two channel case, performing the differentiation leads to the update law

$$
\mathbb{W}_{n+1}=\mathbb{W}_{n}+\mu\left[\begin{array}{cc}
-\alpha_{1} \beta_{1} \gamma_{1} w_{21} & -\alpha_{2} \beta_{2} \gamma_{2} w_{22} \\
\alpha_{1} \beta_{1} \gamma_{1} w_{11} & \alpha_{2} \beta_{2} \gamma_{2} w_{12}
\end{array}\right]
$$

where

$$
\alpha_{i}=4\left(w_{i 1} x_{1}+w_{2 i} x_{2}\right)^{3}
$$

$$
\begin{aligned}
\beta_{i} & =\left(\begin{array}{ll}
-x_{1} w_{i 1} r_{12}-x_{1} w_{2 i} \sigma_{2}^{2}+x_{2} w_{1 i} \sigma_{1}^{2}+ \\
\left.w_{2 i} x_{2} r_{12}\right)
\end{array}\right. \\
\gamma_{i} & =1 /\left(w_{i 1}^{2} \sigma_{1}^{2}+2 w_{i 1} w_{2 i} r_{12}+w_{2 i}^{2} \sigma_{2}^{2}\right)^{3} \\
W & =\left[\begin{array}{ll}
w_{11} & w_{12} \\
w_{21} & w_{22}
\end{array}\right]
\end{aligned}
$$

and $\sigma_{1}^{2}=\mathrm{E}\left\{y_{1}^{2}\right\}, \sigma_{2}^{2}=\mathrm{E}\left\{y_{2}^{2}\right\}, r_{12}=\mathrm{E}\left\{y_{1} y_{2}\right\}$. In true implementation, knowing the actual values of $\sigma_{1}^{2}, \sigma_{2}^{2}$, and $r_{12}$ a priori is not possible but these may be replaced by simple autoregressive estimators of the form

$$
\begin{aligned}
\hat{\sigma}_{i}^{2} & =\lambda \hat{\sigma}_{i}^{2}+(1-\lambda) y_{i}(k)^{2} \\
\hat{r}_{12} & =\lambda \hat{r}_{12}+(1-\lambda) y_{1}(k) y_{2}(k)
\end{aligned}
$$

where $\lambda$ is the estimator's forgetting factor. It should also be noted that a scaling factor is incorporated into the algorithm, since kurtosis is a scale invariant quantity.

The critical assumption here is that the kurtosis of two mixed voice signals has a lower kurtosis than the individual kurtosis values (as hinted at by (4)). However, in the strict sense, (4) is not necessarily always true for speech signals due to the temporal correlation of speech. There is much evidence derived from studies with sampled speech that this relation in (4) often holds true. This issue is addressed in the next section.

\section{SPEECH, SIRPS, AND MIXTURE KURTOSES}

The underlying assumption for the proposed speech signal separation technique is that mixtures of speech signals have a kurtosis lower than the kurtosis values of the individual speech signals. In this section we offer evidence which supports this assumption based on actual speech signals and statistical models for speech.

Eight individual speech signals were sampled and the kurtoses computed. In addition, the kurtosis of a mixture $\left(x_{0}=a s_{0}+(1-a) s_{1}\right)$ of the speech signals was also computed. The results are shown in Figure 2 (solid line). We note that for the $50 \%-50 \%$ mixture $(\mathrm{a}=0.5)$, the individual speech signals are higher in kurtosis than the mixture for $93 \%$ of the studied cases. This indicates that the speech signals will begin to separate from the mixture based on the kurtosis maximization algorithm. As separation proceeds, some of these mixtures may cease further separation (as defined by power ratios), as indicated by the lower probability of both indivudual kurtosis being higher than the mixture kurtosis (Figure 2). However, we have observed in our experiments that there is always one speech signal that is higher in kurtosis than the mixture, indicating that at least this one speech signal could be separated from the mixture; the remaining speech signal might be subsequently separated from residual signal analysis and processing.

Historically, speech probability density functions (PDFs) have been modeled with either the Laplace or Gamma PDF [14] [15]. For these two PDFs, it can be shown that the kurtosis of the sum of these PDFs is lower than the kurtosis values of the individual PDFs. More recently, refinement in the speech model has been achieved through the use of Spherically Invariant Random Processes (SIRP's) also known as circularly or spherically symmetric random processes [16]. 
The use of SIRP's to model speech signals is based on the facts that many random processes are SIRP's including those with Laplace and Gamma PDFs and that actual speech bivariate PDFs (three dimensional amplitude histogram taken from samples of speech signals spaced $t<5 \mathrm{~ms}$ apart) have been shown to exhibit SIRP-like qualities [18], [19], [17]. Based on work by Brehm, the SIRP which includes the Gaussian, Laplace, Gamma, and $K_{0}$ PDF's, was found to be especially suited to modeling the measured densities of speech signals. By continuous variation of $b_{1}$ and $b_{2}$, a whole family of modeling PDF's may be generated, one of which may be a better approximation to the speech PDF than Laplace or Gamma PDFs. Brehm and Stammler found that speech PDFs can be closely matched by choosing

$$
-0.4 \leq b_{1} \leq-\frac{1}{3}
$$

and

$$
b_{2} \geq 0.25
$$

Furthermore, when the time shift between the two speech signals used to compute the bivariate speech PDF is less than $3.8 \mathrm{~ms}$, the bivariate PDF corresponding to SIRP fits the observed contour lines in the bivariate PDF very well [17].

Five SIRPs were modeled according [17] and the Kurtoses computed. In addition the kurtosis of a mixture $\left(a \operatorname{SIRP}_{X}+\right.$ $(1-a)$ SIRP $\left._{Y}\right)$ of the SIRP's was also computed. The results are shown in Figure 2 (dashed line). In all 50\%-50\% mixture cases, $\kappa_{\mathrm{S}_{0}}, \kappa_{\mathrm{S}_{1}}>\kappa_{\mathrm{X}_{1}}$ the individual SIRP's are higher in kurtosis than the mixture meeting the assumed necessary conditions for the proposed algorithm.

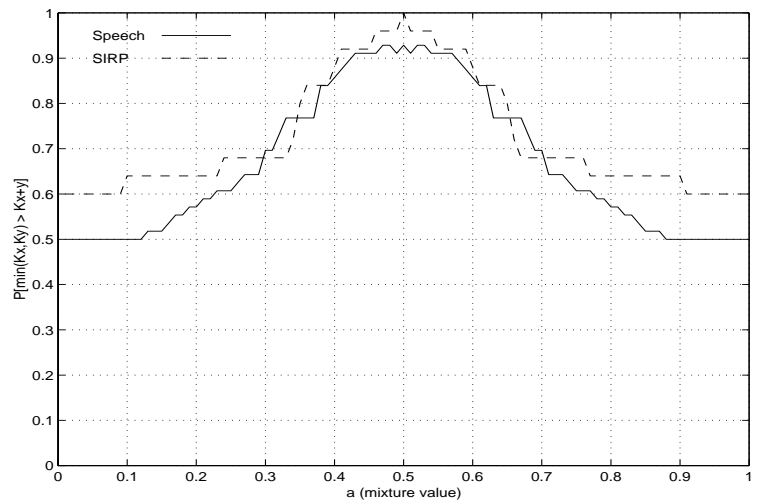

Figure 2.

\section{SEPARATION EXAMPLES}

In this section the performance of the algorithm in (8) is demonstrated using two separtation examples (case I and case II). Here, the mixing matrices are arbitrarily chosen.

\subsection{Case I}

$$
\mathbb{A}_{I}=\left[\begin{array}{ll}
0.7 & 0.3 \\
0.3 & 0.7
\end{array}\right]
$$

$s_{0}$ and $s_{1}$ are sampled speech of a male speakers reading different text segments of a text book with $\kappa_{\mathrm{S}_{0}}=14.9$ and

\begin{tabular}{|l|l|}
\hline stepsize & $\mu=10^{-6}$ \\
moment est. forgetting factor & $\lambda=0.999$ \\
separation matrix initialization & $\mathbb{W}(0)=\left[\begin{array}{cc}1 & 0.1 \\
0.1 & 1\end{array}\right]$ \\
initial moment estimates & $\hat{r}_{12}(0)=0.1$ \\
& $\hat{\sigma}_{1}(0)=0.1$ \\
& $\hat{\sigma}_{2}(0)=0.1$ \\
\hline
\end{tabular}

\section{Table 1. Example Simulation Parameters}

$\kappa_{\mathrm{S}_{1}}=14.2$. The received mixtures $x_{0}$ and $x_{1}$ have kurtosis values of $\kappa_{\mathrm{x}_{0}}=11.6$ and $\kappa_{\mathrm{x}_{1}}=11.1$ verifying the assumption on mixed speech signals having lower kurtosis. The severity of the mixing renders to signals $x_{0}$ and $x_{1}$ unintelligible.

Plotted below are the power ratios of the $s_{0}$ and $s_{1}$ components in both $y_{0}$ and $y_{1}$ which provides a measure of the separation of the source components are the output. The adaptation parameters are found in Table 1.

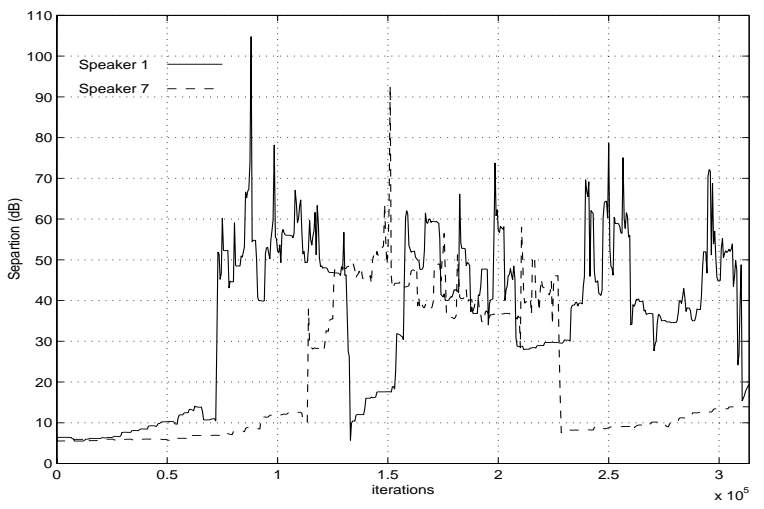

Figure 3. Case I of Source Separation using Algorithm

The achieved separation is very good, exceeding $40 \mathrm{~dB}$ on both channels at times. Qualitatively, listening to the resulting separated signals, the second speech signal was virtually imperceptible.

\subsection{Case II}

Here $s_{0}$ and $s_{1}$ are different speakers reading text segments of a text book with $\kappa_{\mathrm{S}_{0}}=27.9$ and $\kappa_{\mathrm{s}_{1}}=11.3$. The mixing matrix

$$
\mathbb{A}_{I I}=\left[\begin{array}{ll}
0.8 & 0.2 \\
0.7 & 0.3
\end{array}\right]
$$

is used resulting in received mixtures $x_{0}$ and $x_{1}$ have kurtosis values of $\kappa_{\mathrm{x}_{0}}=24.9$ and $\kappa_{\mathrm{x}_{1}}=20.8$. Note here that one mixture violates the assumption that the necessary assumption and only one source is separted. This is thought to be due to the widely disparate source kurtoses.

Again, the power ratios of source components are plotted using the algorithm with the same parameters. The achieved separation on one output is very good, again exceeding $40 \mathrm{~dB}$ on one channel. However, at the other output channel little separtation is acheived (about constant at $10 \mathrm{~dB})$. But, since excellent separation has been acheived at 


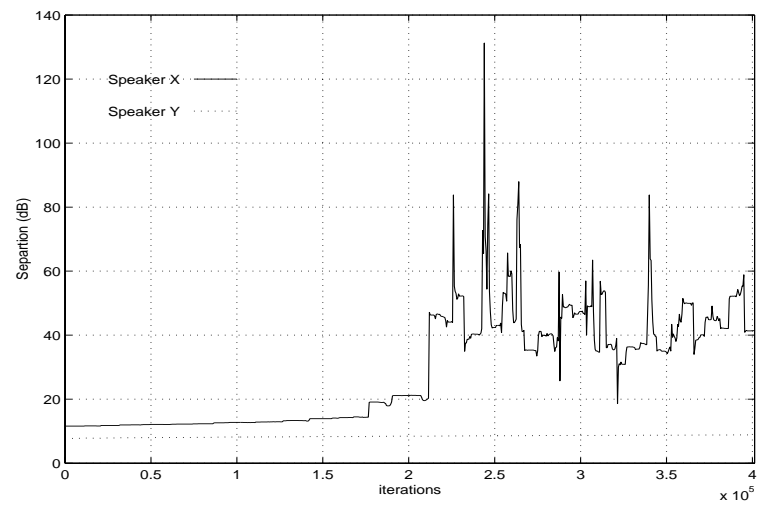

Figure 4. Case II of Source Separation using Algorithm

the other output, it is possible to perform further processing using residual signal analysis.

\section{CONCLUSION}

We have adopted some ideas and results from the digital communications community used in the blind source separation and blind equalization problem and modified them for use in the speech separation problem. The presentation of an algorithm along with its motivation is described through the concept of kurtosis maximization with leptokurtic signals. While originally based on heuristics, the analysis of SIRP's in relation to statistical speech models provides a strong framework for analysis. Initial analysis supports the conjecture regarding speech mixing and kurtosis effects for reasonable known SIRP modeling bounds on human voice.

\section{REFERENCES}

[1] E. Moreau, O. Macchi, "New self-adaptive algorithm for source separation based on contrast functions," Proc. IEEE Signal Processing Workshop on Higher Order Statistics, Lake Tahoe, CA, 1993, pp.215-219.

[2] J. -L. Lacoume, P. Ruiz, "Source Identification: A solution based on the cumulants," Proc. $4^{\text {th }}$ ASSP Workshop Spectral Estimation Modeling, Minneapolis, MN, Aug. 1988, pp.199-203.

[3] L. Castedo, O. Macchi, "Maximizing the Information Transfer for Adaptive Unsupervised Source Separation," Proc. IEEE Signal Processing Advances in Wireless Communications Workshop, Paris, 1997, pp.6569.

[4] P. Comon, "Independent Component Analysis, A New Concept?," Signal Processing, vol. 36, pp.287-314, April, 1994.

[5] J. P. LeBlanc, I. Fijalkow, C.R. Johnson, Jr., "CMA Fractionally Spaced Equalizers: Stationary Points and Stability under IID and Temporally Correlated Sources," International Journal of Adaptive Control and Signal Processing, (accepted for publication).

[6] D. N. Godard, "Self-Recovering Equalization and Carrier Tracking in Two-Dimensional Data Communication Systems," IEEE Trans. on Commun., vol. COM28, No. 11, Nov. 1980.
[7] D.L. Donoho, "On Minimum Entropy Deconvolution," Applied Time Series Analysis, D.F. Findley, Ed., New York: Academic Press, 1981.

[8] J.R. Treichler, M. G. Agee A New Approach to Multipath Correction of Constant Modulus Signals IEEE Trans. on Acoustics, Speech, and Signal Processing April 1983

[9] J. -F. Cardoso, "Source separation using higher order moments," Proc. ICASSP 89, Glasgow, Scotlant, May 1989, vol. 4, pp.2109-2112.

[10] Y. Cao,S. Sridharn, M. Moody, "Multichannel Speech Separtation by Eigendecomposition and Its Application to Co-Talker Interference Removal," IEEE Trans. on Speech, and Audio Proc. vol. 5, No. 3, May 1997.

[11] S. Shamsunder, G. B. Giannakis, "Multichannel Blind Signal Separtation and Reconstruction," IEEE Trans. on Speech, and Audio Proc. vol. 5, No. 6, Nov. 1997.

[12] J. -F. Cardoso, "Iterative technique for blind source separtation using only fourth order cumulunts," Proc. EUSIPCO 92, Brussels, Belgium, Aug. 1992, vol. 2, pp.739-742.

[13] M. G. Bulmer, Principles of Statistics, New York: Dover Publications, 1967.

[14] Davenport, W. B An experimental study of speechwave probability distributions Journal of the Acoustical Society of America, vol. 24, pp. 390-399, Jul. 1952

[15] Paez, M. D. and Glisson, T. H. Minimum mean squared-error quantization in speech IEEE Transactions on Communications, vol. Com-20, pp. 225-230, Apr. 1972

[16] Papoulis, A. Probability, Random Variables, and Stochastic Processes New York, NY: McGraw-Hill, 1989

[17] Brehm, H. and Stammler, W. Description and generation of spherically invariant speech-model signals Signal Processing, vol. 12, no. 2, pp. 119-141, Mar. 1987

[18] Wolf, D. and Brehm, H. Experimental studies on oneand two-dimensional amplitude probability densities of speech signals in Proceedings of the 1973 International Symposium on Information Theory, pp. B 4-6

[19] Brehm, H. Description of Spherically Invariant Random Processes by Means of G-Functions in Lecture Notes in Mathematics (A. Dold and B. Eckmann, Eds.). Springer-Verlag, 1982, vol. 969, pp. 39-73 